\title{
UNSTEADY HEAT TRANSFER IN AN ANNULAR PIPE, PART II: SWIRLING LAMINAR FLOW
}

\author{
KELVIN H.C.S. ${ }^{1}$, YouSIF A.A. ${ }^{1}$ AND ANDREW C. ${ }^{2}$ \\ ${ }^{I}$ Department of Mechanical, Materials and Manufacturing Engineering, \\ ${ }^{2}$ Department of Civil Engineering, \\ Faculty of Engineering, The University of Nottingham, Semenyih, Malaysia \\ choon-seng.ho@nottingham.edu.my,yousif.abakr@nottingham.edu.my, \\ Andy.Chan@nottingham.edu.my
}

\begin{abstract}
The heat transfer problem in magnetocaloric regenerators during magnetization has been described and investigated for convective heat transfer by means of axial flow in part I of this series. This work will focus on enhancing the unsteady heat transfer using swirling laminar flow generated using axial vanes. The governing parameters for this study are, the $\mathrm{D}^{*}$ ratio (Inner diameter/Outer diameter) and the swirl number, S. The study is conducted using dimensional analysis and commercial CFD codes provided by ANSYS CFX. The hydrodynamics and the heat transfer of the model are compared with data from similar cases found in literature and is found to be in the vicinity of good agreement.
\end{abstract}

\begin{abstract}
ABSTRAK:
ABSTRAK: Masalah pemindahan haba semasa pemagnetan dalam penjana semula magnetocaloric telah dikaji untuk pemindahan haba berolak melalui aliran paksi dalam bahagian I siri ini. Kerja ini akan memberi tumpuan kepada peningkatan pemindahan haba tak mantap menggunakan aliran lamina pusaran yang dijana menggunakan ram paksi. Parameter bagi kajian ini, nisbah D * (diameter Inner / diameter Luar) dan nombor pusar, S. Kajian ini dijalankan menggunakan analisis dimensi dan komersial CFD kod yang disediakan oleh ANSYS CFX. Sifat-sifat hidrodinamik dan pemindahan haba di dalam model telah dibandingkan dengan data daripada kes-kes kajian sebelumnya. Kajian ini menemui hasil-hasil yang serupa seperti kajian-kajian yang sebelumnya.
\end{abstract}

KEYWORDS: annular ducts; unsteady heat transfer; magnetic refrigeration/cooling; swirling laminar flow; dimensional analysis

\section{INTRODUCTION}

Undoubtedly, the field of heat transfer has been celebrated by its diversity, applications and distinguished peers. Max Jakob is among the very few whom has not only dedicated his life towards heat transfer research ,but, at the same time inspired and touched the hearts of many [1]. Heat transfer enhancement techniques has been extensively researched into throughout the years, but, as new discoveries enhancements in both science and technology takes place, this will undoubtedly bring heat transfer research into new frontiers (e.g. convective heat transfer enhancement through the use of nanosized fluid particles [2]). Although a wide range of geometries and configurations exist, but, the heat transfer enhancement techniques used are in general common, namely: active, passive and compound enhancement techniques. Active and passive techniques are differentiated by the needs for external power. The former requires an external power 
Kelvin et al.

source to maintain its heat transfer enhancement capability while the latter doesn't. As mentioned earlier, active techniques require an active power or energy source to sustain its capability (e.g. mechanical or electrical aids, surface of fluid vibration, injection or suction forces etc). Passive techniques aim to enhance the heat transfer through either modifying or treating the heat transfer surface (e.g. roughened, treated, extended surfaces etc) or by modifying the flow (e.g. axial, radial vanes etc). Finally, the use of one or more combination of techniques from active and/or passive techniques yield compound techniques (e.g. a roughened surface with swirlers).

This work will focus on the utilization of swirling flow in a concentric annular duct with the aim of enhancing the heat transfer between fluids and solid (In other words, the heat transfer coefficient of the inner wall). Swirling flows in concentric annular ducts can be produced by both active and passive techniques. The distinction between both techniques lies on whether external power is required to generate the swirl phenomenon. In general, swirling flows can be categorized into three distinct groups: Curved, rotating and vortex/decaying flows [3]. Curved flows are created by coil inserts, twisted tapes, helical vanes etc, the idea of this technique is to modify the path of flow on either the inner tube, outer tube or additional inserts to guide the flow along a distinct path. As a result or the modified path along the flow region, curved flows tend to be continuous swirling flows. Rotating flows however are among one of the active techniques and this type of swirling flow is created by either rotating the inner or the outer section of the annular duct. Lastly, vortex flows or swirling decaying flows are usually created by means of tangential inlet, radial or axial canes, twisted tape inserts etc. The idea of this type of configuration is to modify the flow pattern at the inlet of the annular duct and once the flow is swirled, it will then decay out of the system (This means that the tangential velocity decreases along the length of the annulus). The rate of decay depends on the physical properties, hydrodynamic properties, swirl creating method etc.

The use of swirling flow as a means of heat transfer enhancement can be traced back to the work done by Joule [4] on the heat transfer in an annulus using swirling flow(curved flow) created by wire inserts. Work on swirling laminar flow in concentric annular ducts can be found in the work done by Farias Neto et al. [5]

After a perusal of the overwhelming literature, heat transfer in concentric annular ducts is still far from being understood especially under unsteady heat flux conditions. Part I of this series of papers has briefly described the problem and investigated for axial flows. In this paper, dimensional analysis will be incorporated as a means of preliminary analysis before numerical analysis is done for swirling flows in the laminar region.

\section{MODEL DESCRIPTION}

This section will unveil the details of the numerical model. This information includes a description of the model, boundary conditions and transient parameters, a description of relevant materials and general governing equations, numerical methods used and lastly a description on the verification and validation of the model. 
Kelvin et al.

\section{NUMERICAL MODEL DESCRIPTION}

After defining the problem in the previous section, the problem is then investigated numerically using commercial CFD codes. Figure 1 shows the 3D schematic of a concentric annular duct with a solid core.

(a)

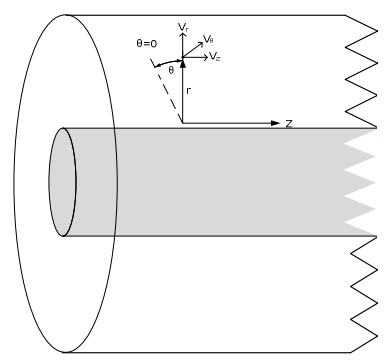

(b)

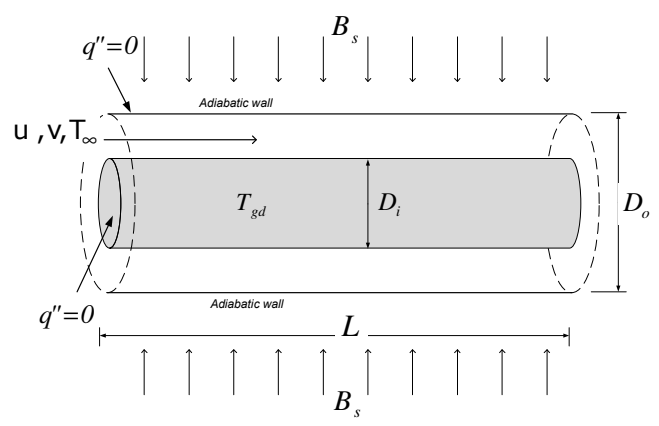

Fig.1: Physical model of problem: (a) Cylindrical coordinate system; (b) Concentric annular duct.

\subsection{Numerical Model}

Figure 1(a) shows the definition of the coordinate system used by the model and Fig. 1(b) shows the $3 \mathrm{D}$ schematic of a concentric annular duct with a solid core. The model consists of two parts, core and fluid domain. Both domains are assumed to compose of isotropic and homogenous material. The core domain is made of gadolinium metal while the fluid domain is made of water. The length of the model is set to $70 \mathrm{~mm}$ and the inner diameter and outer diameter is govern by the $D^{*}$ ratio that varies between $1 / 8,1 / 4,1 / 2$ and $3 / 4$ with the outer diameter remained at $40 \mathrm{~mm}$.

Figures 2(a)-(e) show the mesh used to discretize the model. Figures 2(a)-(d) show the top view of the model for all $D^{*}$ ratios and Fig. 2(e) shows the side view of the model. The total nodes used for each case is approximately $(500,000 \pm 50,000)$. In order to improve boundary layer approximations, especially the fluid-solid interface, the boundary has been inflated with layers of nodes and this is depicted in Fig. 3(a)-(b). The layers of inflation vary between 10-15 for the outer wall and inner wall. It should be noted that the boundary at the inner wall is divided in both fluid and solid near wall layers. 
Kelvin et al.

(a)

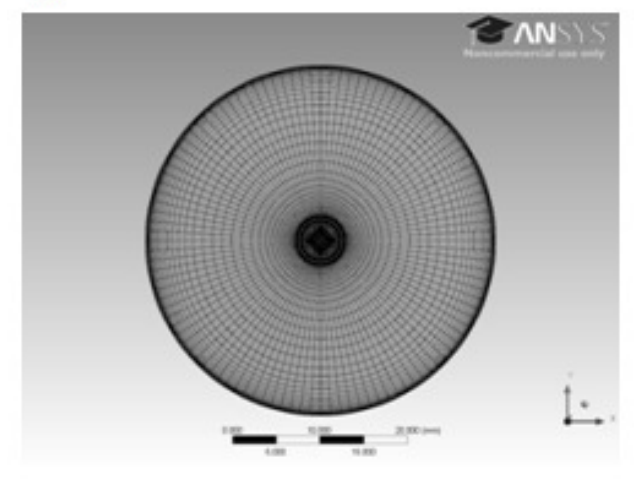

(c)

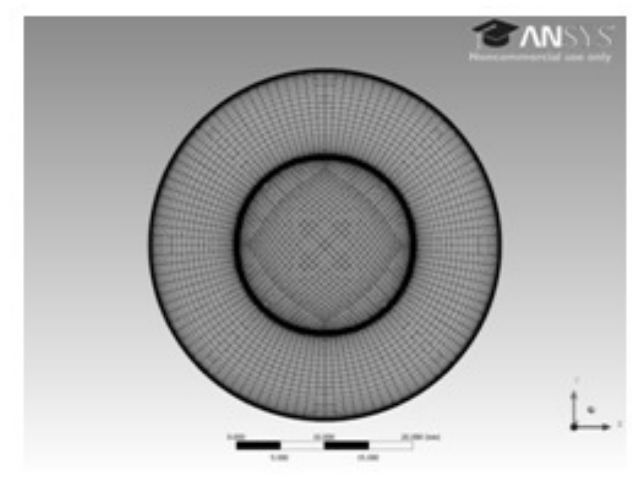

(b)

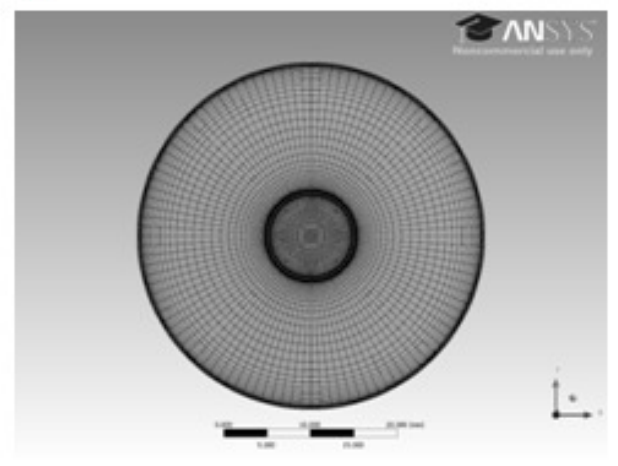

(d)

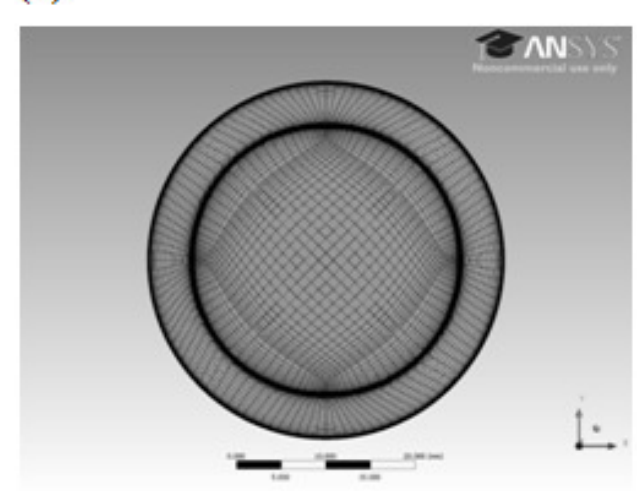

(e)

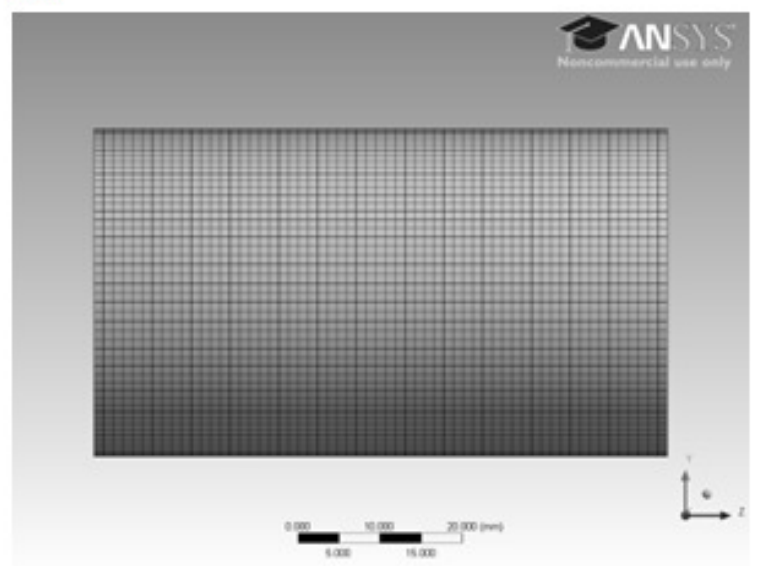

Fig. 2: Semi-structured meshes: (a) $D^{*}=1 / 8$; (b) $D^{*}=1 / 4$; (c) $D^{*=1 / 2}$; (d) $D^{*=3 / 4}$; (e) Side view of mesh. 
(a)

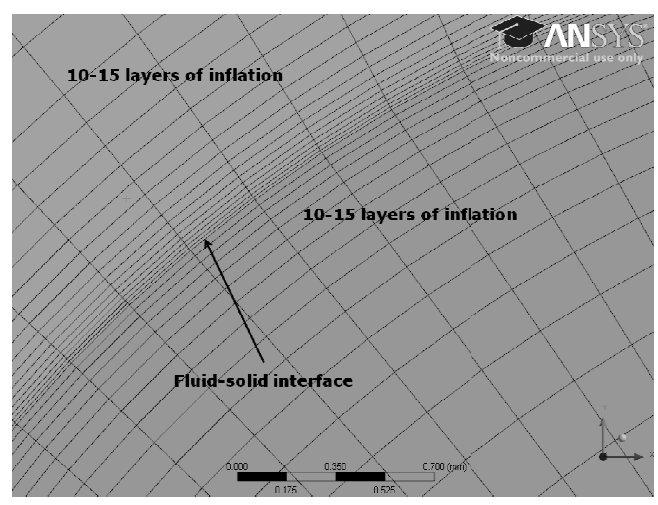

(b)

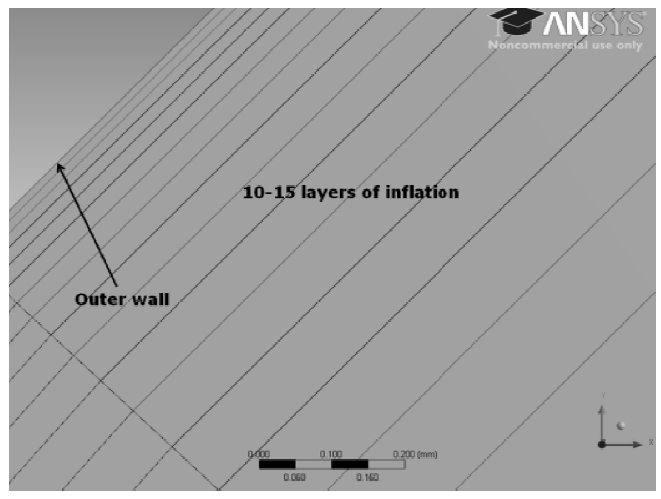

Fig. 3: Inflation layers at boundary: (a) Fluid-solid interface; (b) Outer wall of fluid domain.

Since the main aim of this study is to obtain an understanding on the unsteady heat transfer in an annular duct for disturbed swirling laminar flows. Dimensional analysis will be used as a preliminary tool to identify governing parameters. Assuming that the wall heat transfer coefficient is a function of the following parameters;

$$
h\left(D_{o}, D_{i}, L, \rho_{f}, \mu_{f}, k_{f}, c_{f}, U_{\infty}, V_{\infty}\right)
$$

If the primary measuring units is chosen to be mass, length, time and temperature $(M$, $L, T$ and $\theta$ ), then, according to the Buckingham $\Pi$-theorem[6] we will have $(10-4=6) 6$ independent dimensionless relations.

The dimensionless quantities are selected a follows:

Nusselt number: ${ }^{N u=\frac{h L}{k_{f}}}$

Reynolds number: $\operatorname{Re}_{L}=\frac{\rho_{f} U_{\infty} L}{\mu_{f}}$

Prandtl number: $\operatorname{Pr}=\frac{\mu_{f} c_{f}}{k_{f}}$

Diameter ratio: $D^{*}=\frac{D_{i}}{D_{o}}$

Aspect ratio: $A^{*}=\frac{D_{o}-D_{i}}{L}$

Swirl ratio: $S=\frac{V_{\infty}}{U_{\infty}}$

Hence,

$$
h=\frac{k_{f}}{\left(D_{o}-D_{i}\right)} \Psi\left(\operatorname{Re}, \operatorname{Pr}, D^{*}, A^{*}, S\right)
$$

The swirl ratio defined here is similar to that of Zhang et al. [7], but for simplicity the simpler version is opted. 
Kelvin et al.

It is assumed that the effect of $D^{*}$ and $S$ would be exceedingly significant compared to that of $A^{*}$ and we know that the wall heat transfer coefficient is a function of the Reynolds number and the Prandtl number, thus, the governing parameters of this study are: (1) $D^{*}$ ratio and (2) $S$ number. The variation in the $D^{*}$ ratio will provide us with a thorough investigation from very thin concentric annular duct to near cylindrical duct configuration.

Next, dimensional analysis will be used to identify the governing parameters for the wall heat flux. Assuming that the wall heat flux is a function of the following parameters;

$$
\mathrm{q}^{\prime \prime}\left(\mathrm{D}_{\mathrm{i}}, \mathrm{L}, \mathrm{a}_{\mathrm{s}}, \mathrm{k}_{\mathrm{s}}, \mathrm{h}, \Delta \mathrm{T}, \mathrm{t}\right)
$$

If we were to choose the same primary units as before, we will have (8-4=4) 4 independent dimensionless relations.

The dimensionless quantities are selected a follows:

Biot number: $B i=\frac{h D_{i}}{k_{s}}$

Fourier number: $\quad$ Fo $=\frac{\mathrm{a}_{\mathrm{s}} \mathrm{t}}{\mathrm{D}_{\mathrm{i}}^{2}}$

Aspect ratio: $\frac{\mathrm{D}_{\mathrm{i}}}{\mathrm{L}}$

Dimensionless form of Fourier law: $\frac{q^{\prime \prime} D_{i}}{k_{s} \Delta T}$

Hence,

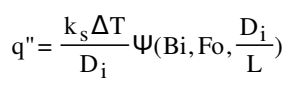

\subsection{Material Properties and Governing Equations}

The incompressible Newtonian fluid used as heat exchanging fluid is water with constant physical properties (Table 2). The properties of gadolinium are summarized in Table 2 and are assumed to be at a constant value throughout the numerical study. The non-linear partial equations that govern the conservation of mass, momentum, energy and turbulent energy are shown in equations (3-8).

Mass Conservation $(\mathrm{r}, \theta, \mathrm{z})$ :

$$
\frac{\partial v_{r}}{\partial r}+\frac{v_{r}}{r}+\frac{1}{r} \frac{\partial v_{\theta}}{\partial \theta}+\frac{\partial v_{z}}{\partial z}=0
$$

Momentum Conservation $(\mathrm{r}, \theta, \mathrm{z})$ :

$$
\begin{aligned}
& \rho\left(\frac{\partial v_{r}}{\partial t}+v_{r} \frac{\partial v_{r}}{\partial r}+\frac{v_{\theta}}{r} \frac{\partial v_{r}}{\partial \theta} \frac{v_{\theta}^{2}}{r}+v_{z} \frac{\partial v_{r}}{\partial z}\right)= \\
& \frac{\partial P}{\partial r}+\mu\left(\frac{\partial^{2} v_{r}}{\partial r^{2}}+\frac{1}{r} \frac{\partial v_{r}}{\partial r} \quad \frac{v_{r}}{r^{2}}+\frac{1}{r^{2}} \frac{\partial^{2} v_{r}}{\partial \theta^{2}} \frac{2}{r^{2}} \frac{\partial v_{\theta}}{\partial \theta}+\frac{\partial^{2} v_{r}}{\partial z^{2}}\right)
\end{aligned}
$$




$$
\begin{gathered}
\rho\left(\frac{\partial v_{\theta}}{\partial t}+v_{r} \frac{\partial v_{\theta}}{\partial r}+\frac{v_{\theta}}{r} \frac{\partial v_{\theta}}{\partial \theta} \quad \frac{v_{\theta} v_{r}}{r}+v_{z} \frac{\partial v_{\theta}}{\partial z}\right)= \\
\frac{1}{r} \frac{\partial P}{\partial \theta}+\mu\left(\frac{\partial^{2} v_{\theta}}{\partial r^{2}}+\frac{1}{r} \frac{\partial v_{\theta}}{\partial r} \quad \frac{v_{\theta}}{r^{2}}+\frac{1}{r^{2}} \frac{\partial^{2} v_{\theta}}{\partial \theta^{2}}+\frac{2}{r^{2}} \frac{\partial v_{r}}{\partial \theta}+\frac{\partial^{2} v_{\theta}}{\partial z^{2}}\right) \\
\rho\left(\frac{\partial v_{z}}{\partial t}+v_{r} \frac{\partial v_{z}}{\partial r}+\frac{v_{\theta}}{r} \frac{\partial v_{z}}{\partial \theta}+v_{z} \frac{\partial v_{z}}{\partial z}\right)= \\
\frac{\partial P}{\partial z}+\mu\left(\frac{\partial^{2} v_{z}}{\partial r^{2}}+\frac{1}{r} \frac{\partial v_{z}}{\partial r}+\frac{1}{r^{2}} \frac{\partial^{2} v_{z}}{\partial \theta^{2}}+\frac{\partial^{2} v_{z}}{\partial z^{2}}\right)_{(6)}
\end{gathered}
$$

Energy Conservation $(\mathrm{r}, \theta, \mathrm{z})$ :

$$
\begin{aligned}
& \rho_{f} c_{p, f}\left(\frac{\partial T}{\partial t}+v_{r} \frac{\partial T}{\partial r}+\frac{v_{\theta}}{r} \frac{\partial T}{\partial \theta}+v_{z} \frac{\partial T}{\partial z}\right)= \\
& k_{f}\left[\frac{1}{r} \frac{\partial}{\partial r}\left(r \frac{\partial T}{\partial r}\right)+\frac{1}{r^{2}} \frac{\partial^{2} T}{\partial \theta^{2}}+\frac{\partial^{2} T}{\partial z^{2}}\right] \\
& \rho_{s} c_{p, s}\left(\frac{\partial T}{\partial t}\right)=k_{s}\left[\frac{1}{r} \frac{\partial}{\partial r}\left(r \frac{\partial T}{\partial r}\right)+\frac{1}{r^{2}} \frac{\partial^{2} T}{\partial \theta^{2}}+\frac{\partial^{2} T}{\partial z^{2}}\right]
\end{aligned}
$$

\subsection{Boundary Conditions and Transient Parameters}

The governing equations in the previous section are subjected to the following boundary conditions. At both walls, the flow is prohibited from slipping and at the same time impermeable $(u=v=0)$. The outer wall is assumed adiabatic while the temperature of the inner wall will vary according to the hydrodynamic parameters of the duct over time. At free stream, both axial and tangential velocity is assumed to approach $U \infty$ and $V \infty$. The flow in the annular duct is assumed steady and uniform throughout the length of the duct. The swirl phenomenon is created as a result of disturbed axial flow by using a set of axial vanes, thus, the flow after the vanes will be two-dimensional (vortex flow or swirling decaying flow).

\subsection{Numerical Computation}

The numerical study is conducted using CFD codes provided by ANSYS CFX and the simulated cases are summarized in Table 1 . The test cases are divided into two groups, the first group will be simulated under constant $\mathrm{S}$ ratio for different $D^{*}$ ratios and different axial velocities. The second group is simulated under fixed axial velocity for different $S$ ratios and $D^{*}$ ratios. As per the pre-analysis done using dimensional analysis, we have determined the governing parameters of this study, thus, the above test cases have been structured and selected for this purpose, whereby, the first group of thes cases would study the effect of the $D^{*}$ ratio and Reynolds number while the second test group will be used to study the effect of different $\mathrm{S}$ ratios. The Barth and Jesperson (high resolution scheme) and a series of structured mesh are used to discretized the equations (3-8). The transient term is discretized using the second order backward Euler scheme. A grid independent test and a time step independent test are conducted on the model [8]. The gird independent test is conducted by comparing between two different node counts $\left(5 \times 10^{5}\right.$ and $\left.10 \times 10^{5}\right)$. The time step independent test is conducted by comparing between two different time steps (300 and 3000). 
Kelvin et al.

\subsection{Verification and Validation}

Tests conducted on the model confirm that the model is indeed grid and time step independent (Discrepancies below $0.1 \%$ ). As a result, $\times 10^{5}$ nodes and 3000 time steps were used throughout the numerical study. Finally, the accuracy of computed numerical results over each node satisfies the RMS convergence criteria of $10^{-12}$.

\section{NUMERICAL RESULTS AND DISCUSSION}

This section presents the numerical results, in the form of plots and is facilitated with a series of discussion. The first section will discuss on the hydrodynamics and heat transfer characteristics. Subsequent sections will focus on the effect of different governing parameters (e.g. $S, D^{*}$ and $R e$ ).

\subsection{On the Hydrodynamic and Heat Transfer Characteristics}

Since it is the nature of vortex flows to exhibit signs of decay along the flow path due to friction, thus, for an annular duct with sufficient hydrodynamic length we would expect to witness a significant decay in the tangential velocity component due to the lack of a continuous force to sustain the tangential flow. Figure 4 depicts the axial and tangential velocity component for one of the cases $\left(D^{*}=0.125, S=1\right.$ and $\left.u=0.01 \mathrm{~m} / \mathrm{s}\right)$ at different axial distances along the duct. Since the flow is still developing, the near wall velocity will tend to decrease as it proceeds further downstream as a result of an increase in the velocity boundary layer until the flow reaches its fully developed state. The hydraulic diameter of this study is defined as the difference between the Outer and inner diameter $\left(D_{0}-D_{i}\right)$ and this is applied consistently throughout the study. A detail discussion on the friction factor, pressure drops and the velocity boundary layer will not be given as it is not within the scope of this paper. Furthermore, the hydrodynamics for both steady and unsteady heat fluxes should be the same assuming that the flow characteristics are independent of temperature.
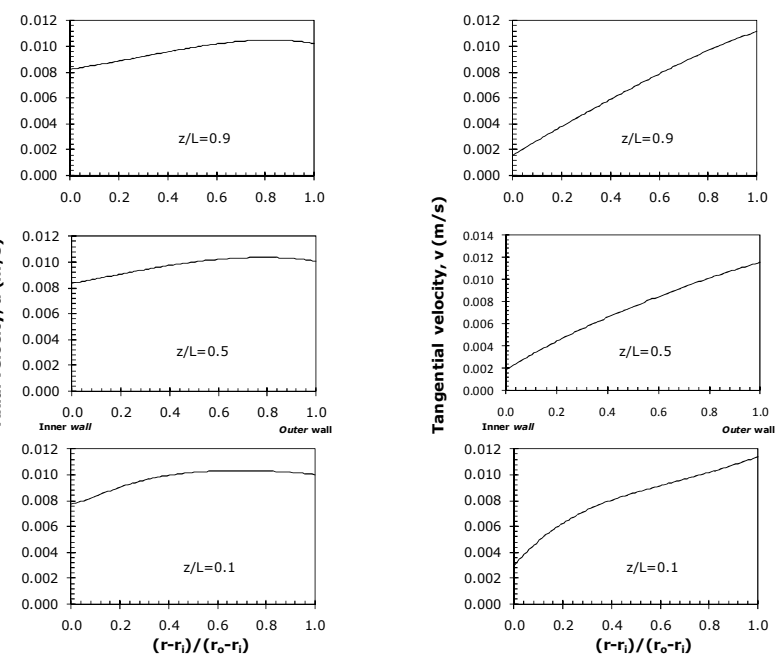

(a)

(b)

Fig. 4: Axial and tangential velocity plots for $D^{*}=0.125, S=1, u=0.01 \mathrm{~m} / \mathrm{s}$. 
Kelvin et al.

For laminar flows, heat transfer through molecular diffusion (molecular conduction) is dominant. Since the flow and solid is assumed to be homogenous and isotropic, longitudinal temperature variation of the solid core will be negligible compared to the radial temperature variation. For heat transfer in circular or annular ducts, experience tells us that the wall heat transfer coefficient will always be proportional but not limited to the Reynolds number $(R e)$ and the Prandtl number $(P r)$ (e.g. $N u \propto P e^{a}$ ), whereby, $P e$ is Peclet number which has a power of $a$. In particular, it is of great interest to understand how the cooling time $\mathrm{t}$ is affected by the governing parameters (e.g. $\mathrm{Nu}$ and $q$ "), at the same time, it is also crucial to investigate how the wall heat flux behaves with respect to different flow and geometrical arrangements over a limited period of time. The Prandtl number of water for this study is chose to be 6.3 which is greater that 1 , thus, according to boundary layer theory the thermal entrance length will be greater than the hydrodynamic entrance length $\left(\delta_{\mathrm{T}} \gg \delta_{\mathrm{V}}\right)$. In addition, for a problem of unsteady heat transfer, we would expect the thermal boundary layer to undergo the developing process and subsequently disappears when the solid reaches the same temperature as its surrounding fluid.

From the previous section, the use of dimensional analysis has yield fruitful relations. We know that the wall heat transfer coefficient is defined by equation (1). The effect of $A^{*}$ towards $\mathrm{h}$ is small compared to the other parameters, thus, $A^{*}$ is removed from the relation, giving;

$$
\left.h=\frac{k_{f}}{\left(\begin{array}{ll}
D_{o} & D_{i}
\end{array}\right)} \Psi_{\left(R e, P r, D^{*}, S\right.}\right)
$$

The application of further in depth knowledge on dimensional analysis, hydrodynamics and heat transfer will provide further insights on the problem, but, for the meantime the definition of $h$ in equation 9 will suffice. Next, a relation for the wall heat flux was also deduced using dimensional analysis (equation (2)). Under steady-heat flux conditions, the wall heat flux would often be a function of the Biot number, geometrical properties and the temperature gradient, but, under unsteady conditions the dimensionless time defined by the Fourier number would be vital. Similar to $A^{*}$, the aspect ratio in the definition of q" may be neglected as well, hence, the simplified definition;

$$
\left.q^{\prime \prime}=\frac{k_{s} \Delta T}{D_{i}} \psi_{(B i, F o}\right)
$$

\subsection{On the Effect of Inlet Velocity}

The inlet velocity is a characteristic of the hydrodynamics of the problem and its effect towards the wall heat transfer coefficient is direct. In this study, the two dimensional flow is assumed created by forcing fluid through a set of vanes which are positioned at a fixed angle on a buff object (e.g. $30^{\circ}, 45^{\circ}$ and $60^{\circ}$ ). For discussion purposes, two different Reynolds number will be used. The first is $R e_{L}$ with was defined during dimensional analysis, and the other $R e_{\text {eff }}$ which is based on the hydraulic diameter such that ( $\left.R e_{e f f}=\frac{\rho_{f} U_{\infty}\left(D_{o} D_{i}\right)}{\mu_{f}}\right) . R e_{L}$ is used as a momentum-viscous ratio that is independent of the diameter and it is used to represent the effect of different inlet velocities without the effect of the hydraulic diameter. Subsequently, $\mathrm{Re}_{\text {eff }}$ takes into account both inlet velocity and the hydraulic diameter and is used for discussion purposes.

Since the heat transfer coefficient is known to be proportional to the Reynolds number or the fluid velocity, thus, an increase in inlet velocity will lead to an increase in the wall 
Kelvin et al.

heat transfer coefficient. This is shown in Fig. 5, whereby the wall heat transfer coefficient is plotted against different Reynolds number within the laminar regime. Each of the line represents a different $D^{*}$ ratio. From the plot, we can conclude that the wall heat transfer coefficient is linearly proportional to the inlet velocity in the laminar regime. Furthermore, an increase in hydraulic diameter also increases the wall heat transfer coefficient and this increase is more significant at higher Reynolds numbers. As a result, we have the following relations;

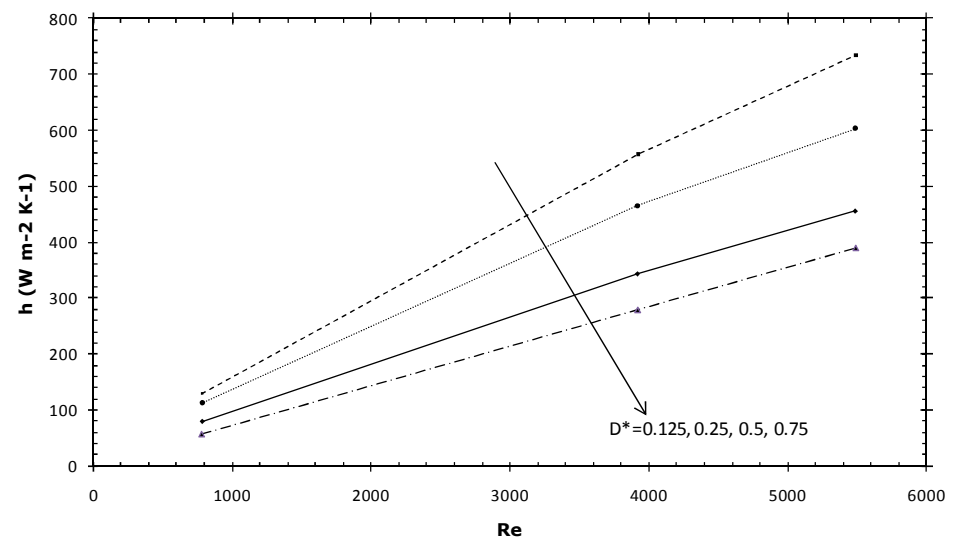

Fig. 5: Variation of wall heat transfer coefficient for different Reynolds number for constant $D^{*}$ ratio.

$$
\begin{gathered}
h \propto U, \\
q^{\prime \prime} \propto h, \\
t \propto \frac{1}{U_{\infty}} .
\end{gathered}
$$

From the axial and tangential velocity plots for $S=1$ in Fig. 6 . The velocity plots are taken at three distinct axial locations along the flow path $(z / L=0.1,0.5$ and 0.9$)$. It is observed that the momentum flux tend is biased towards the outer wall, but, diminishes as the $D^{*}$ ratio increases. In this case, the use of $\operatorname{Re}_{\text {eff }}$ as a comparison medium would be more fruitful since in involves the $D^{*}$ ratio. As the $D^{*}$ ratio increases, the hydraulic diameter, $D_{h}$ decreases hence the effective Reynolds number, $R e_{\text {eff. }}$. So, for very low $R e_{e f f}$ the velocity profile will be uniformly distributed, hence, the uniformity of wall heat transfer coefficient on both inner and outer wall. This phenomenon is tends to happen at higher $R e_{\text {eff }}$ and was also observed by Zhang et al. [9]. As a result the wall heat transfer coefficient on the outer wall will be higher compared to the inner wall.

Although the inlet velocity does not explicitly falls into the relation for wall heat flux, but, a careful inspection eventually reveals its implicit relation to the wall heat flux through the wall heat transfer coefficient, whence, the Fourier's equation for heat transfer coefficient $\left(q^{\prime \prime}=h\left(T_{0}-T_{\infty}\right)\right)$ and can be traced to the times Newton. Both are independent parameters which are linked up by $\Delta \mathrm{T}$. Recalling equation (1), our definition of the wall heat transfer coefficient is a monomial relation with the parameters $R e, D^{*}$ and $S$. The wall heat transfer coefficient will play its role in reducing the temperature gradient between fluid and solid so long as $\Delta \mathrm{T}$ is not NIL. 

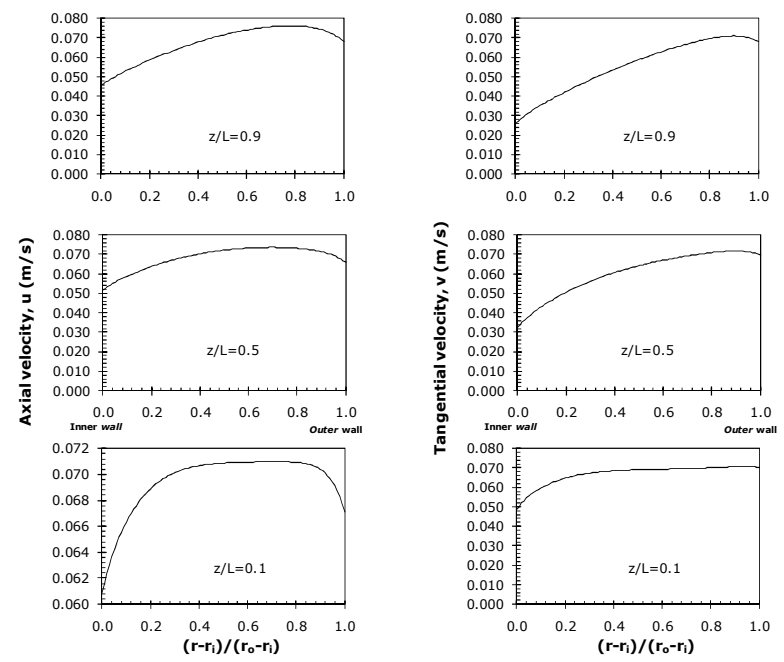

(a)

(b)
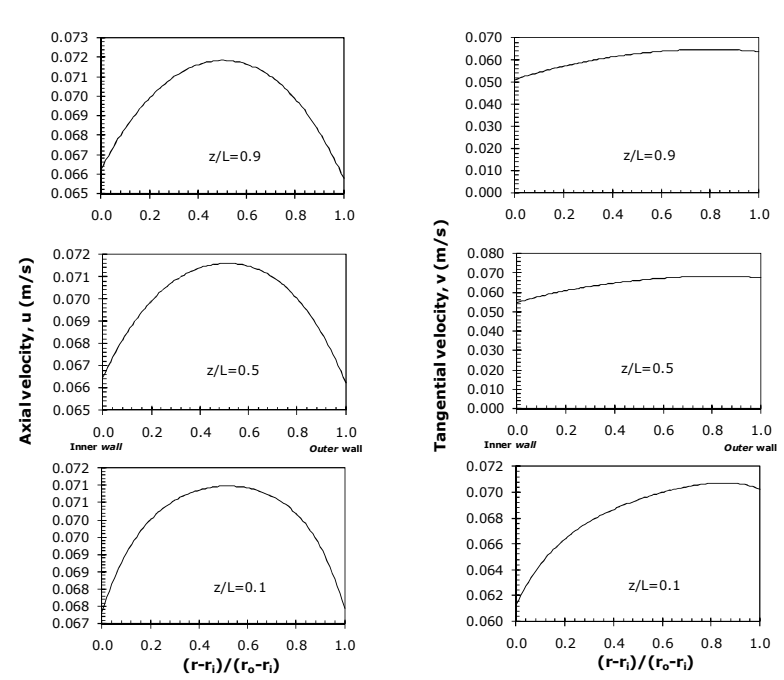

(c)

(d)

Fig. 5: Axial and tangential velocity plots at $z / L=0.1,0.5$ and 0.9 for (a)-(b) $D^{*}=0.25, \mathrm{~S}=1$ and $u=0.07 \mathrm{~m} / \mathrm{s}$ and (c)-(d) $D^{*}=0.75, S=1$ and $u=0.07 \mathrm{~m} / \mathrm{s}$.

\subsection{On the Effect of Geometric Ratio}

From dimensional analysis we have defined two independent ratios that represents the geometrical configuration of the problem and among them, one is negligible and the other a crucial governing parameter. As per our previous discussions, the effect of $D^{*}$ is said to be dominant to that of $A^{*}$ assuming that the radial temperature gradients are dominant, furthermore, since both $D_{o}$ and $L$ are fixed, thus, $D^{*}=\frac{D_{i}}{D_{o}}$ and $A^{*}=\frac{D_{o} \quad D_{i}}{L}$ will present itself under a common purpose under different denominators. As a result, discussion from here on will be made with reference to $D^{*}$ only. 
Kelvin et al.

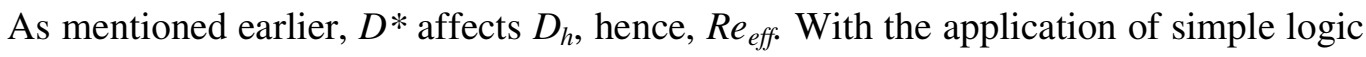
and some knowledge in heat transfer and hydrodynamics we can deduce a simple relation between these variables within the limits of the laminar regime for $S=1$;

$$
h \propto \frac{1}{D^{*}}
$$

These results are shown in Fig. 7 for variations of $h$ with reference to different $D^{*}$ ratios under constant $\operatorname{Re}_{L}$. Observations shows that the nonlinearity between $\mathrm{h}$ and $D^{*}$ increases as the inlet velocity increases (e.g. the number of vortices increases as the flow regime transits from laminar to the transition regime).

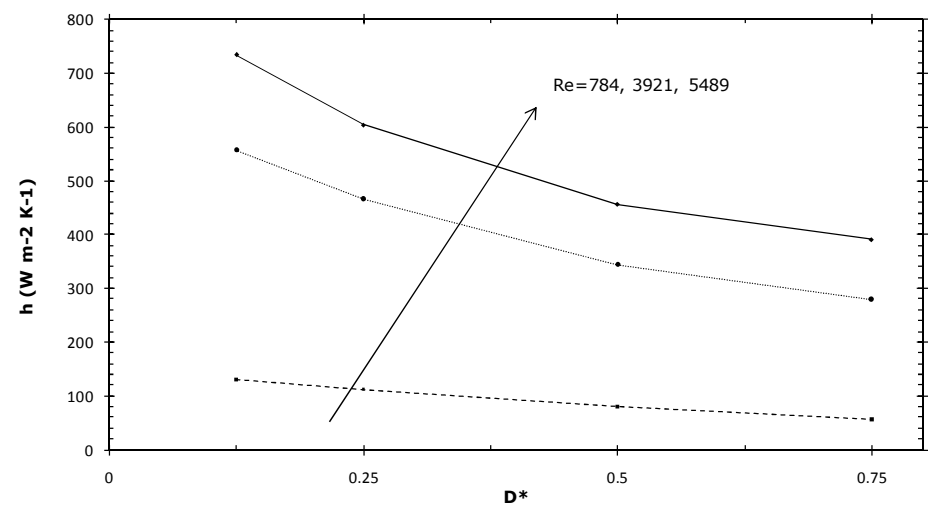

Fig. 6: Variation of wall heat transfer coefficient for different $D^{*}$ ratios for constant Reynolds number.

Within these limits the wall heat transfer coefficient is inversely proportional to the $D^{*}$ ratio and since the wall heat flux is also proportional to the wall heat transfer coefficient;

$$
q^{\prime \prime} \propto h
$$

We can then deduce a crude approximation between time of cooling and the $D^{*}$ ratio;

$$
t \propto D^{*}
$$

From the velocity profiles shown in Fig. 6 and 8, it is observed that as $D^{*}$ increases from 0.125 to 0.75 the uniformity of the axial velocity profiles tend to increase with the disappearance of the bias towards the outer wall, however, this is different for the tangential velocity profile whereby a variation in $D^{*}$ has little effect towards it. Although the tangential component is not sensitive towards $D^{*}$, but, an increase in the inlet velocity is found to increase the uniformity of the tangential profile. This can be seen by comparing Fig. 6 (b) and (d) with Fig. 8 (b) and (f).

\subsection{On the Effect of Flow Ratio}

The flow ratio that is defined in this study is the swirl ratio, $S$ which is measure based on the ratio between the tangential and axial velocity component. The swirl ratio is varied from 0.1 up to 1.5 to investigate its effect on heat transfer. When $S$ is less than $1(S<1)$, the axial velocity component is dominant, whereas, when $S$ is greater than $1(S>1)$ the tangential velocity component is dominant. 


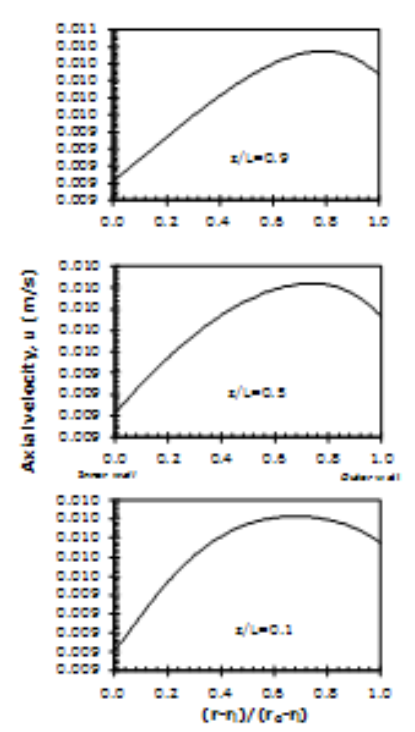

(a)

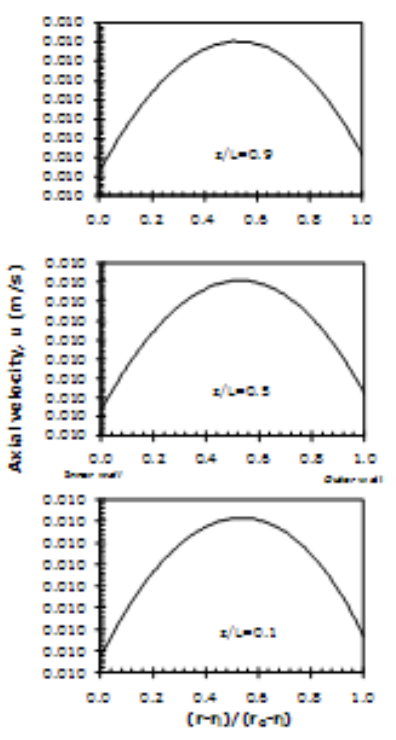

(e)

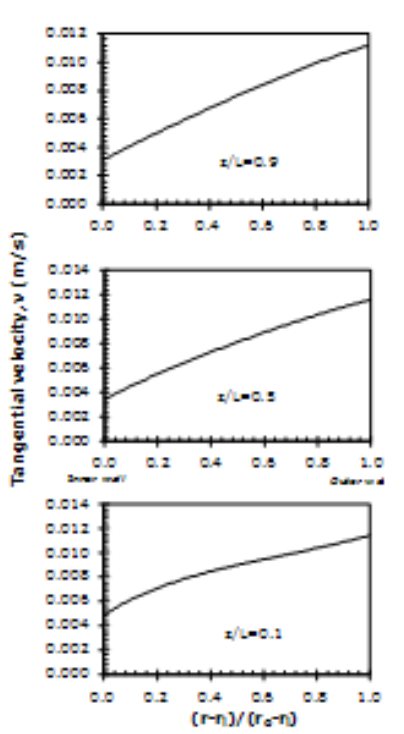

(b)

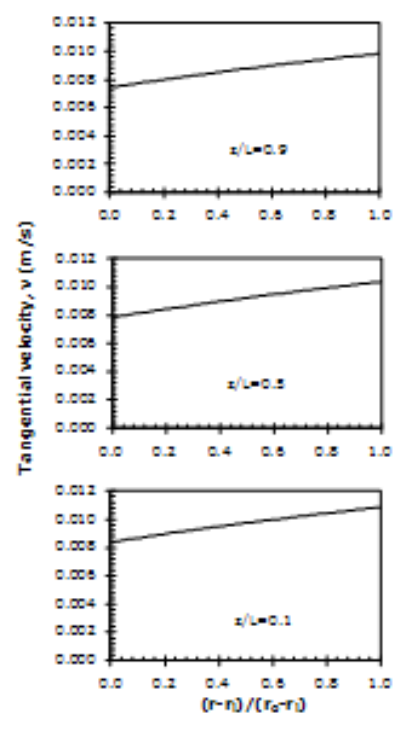

(f)

Fig. 7:. Axial and tangential velocity plots at $z / L=0.1,0.5$ and 0.9 for $u=0.01 \mathrm{~m} / \mathrm{s}$ and $S=1$ (a)-(b) $D^{*}=0.25$, (c)-(d) $D^{*}=0.50$ and (e)-(f) $D^{*}=0.75$.

From Fig. 8 (a),(b) and 10 (a)-(f), we can observe how different $S$ ratios affect the laminar velocity profiles. It is observed that, when $S<0.5$ the axial velocity profile is biased towards the inner wall and as $S$ increases, this bias tend to shift towards the outer wall. A change in the $S$ ratio would affect the magnitude of the tangential velocity profile since $S$ varies because of different tangential velocities. 
From Fig. 9, we know that an increase in $S$ would increase the wall heat transfer proportionally. In addition to that, the wall heat transfer coefficient becomes increasingly sensitive towards a change in $D^{*}$ sensitive when $S$ is above 0.5 . Since an increase in $S$ yields a corresponding increase in $\mathrm{h}$, as a result, the time needed for cooling will also be reduced. Thus, the relations;

$$
\begin{aligned}
& h \propto S, \\
& t \propto \frac{1}{S}
\end{aligned}
$$

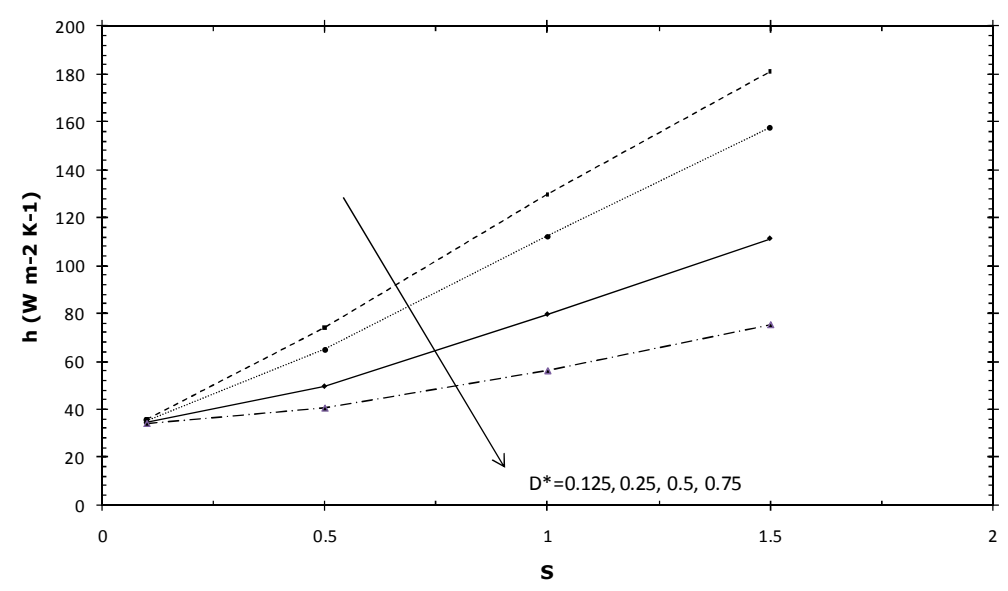

Fig. 8: Variation of wall heat transfer coefficient for different $\mathrm{S}$ ratios for constant $D^{*}$ ratio and $u=0.01 \mathrm{~m} / \mathrm{s}$.
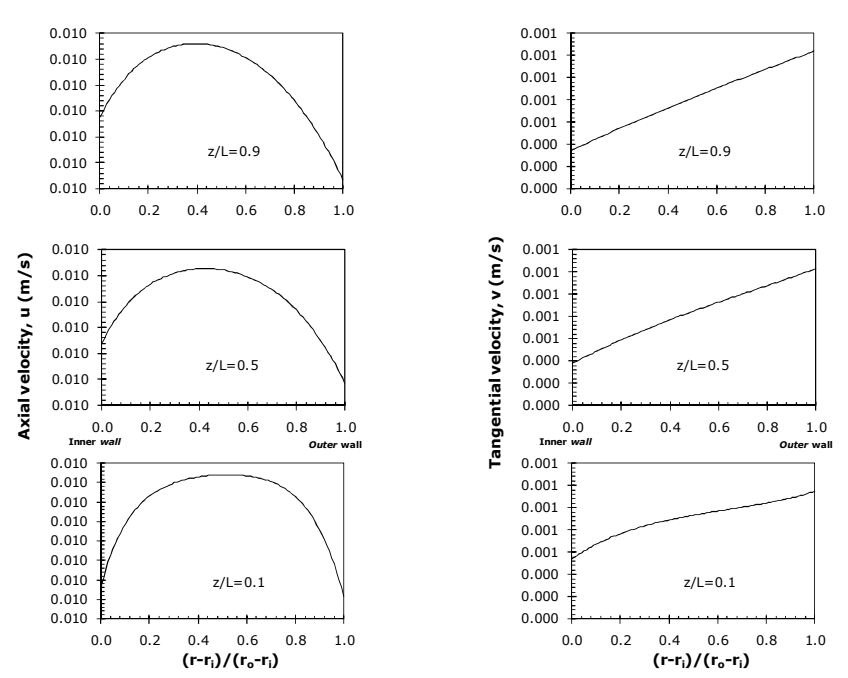

(a)

(b) 
IIUM Engineering Journal, Vol. 12, No. 6, 2011: Special Issue in Science and Ethics

Kelvin et al.
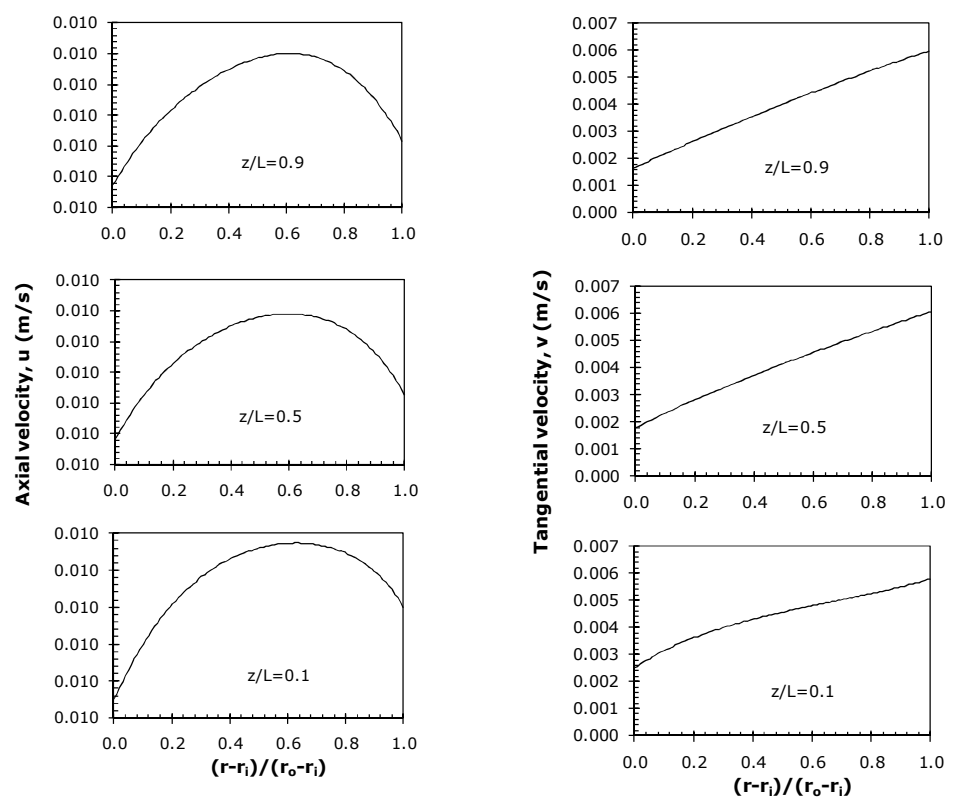

(c)

(d)
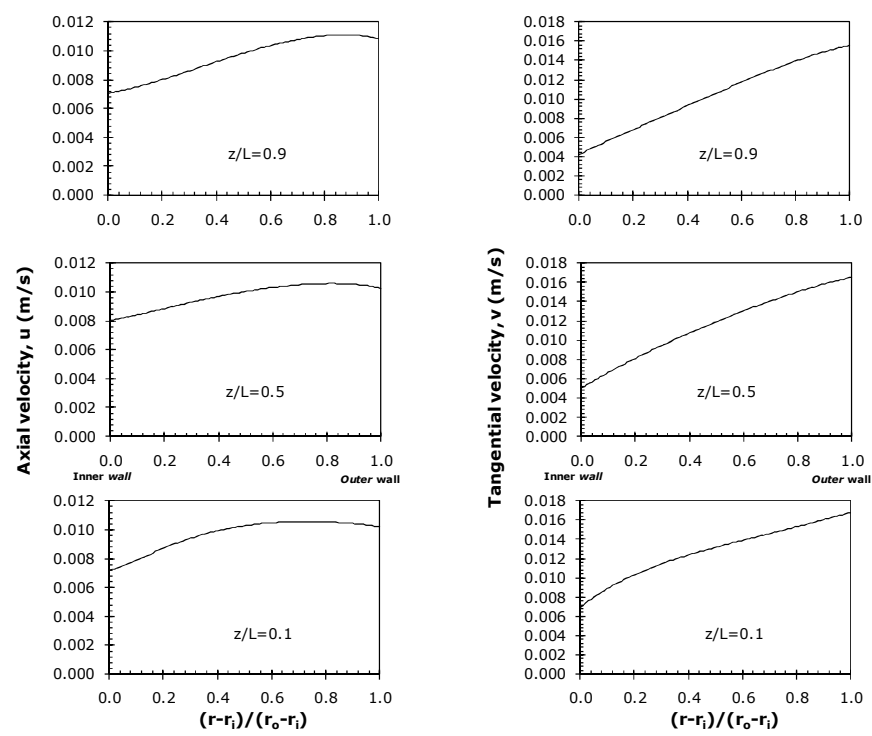

(e)

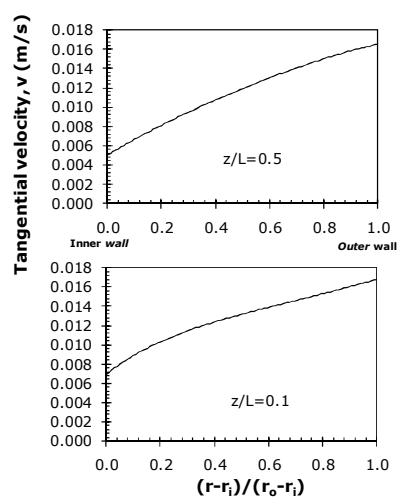

(f)

Fig. 9: Axial and tangential velocity plots at $z / L=0.1,0.5$ and 0.9 for $u=0.01 \mathrm{~m} / \mathrm{s}$ and $D^{*}=0.25$ (a)-(b) $S=0.10$, (c)-(d) $S=0.50$ and (e)-(f) $S=1.50$.

\section{CONCLUSIONS}

The unsteady heat transfer in a concentric annular duct has been investigated using dimensional analysis and commercial CFD codes and the following conclusions can be deduced from the results; 
It can be concluded that;

a. The heat transfer coefficient for unsteady heat fluxes such as the on above can be briefly represented by the relation;

$$
h \propto\left(\operatorname{Re}, \frac{1}{D^{*}}, S\right)
$$

b. The wall heat flux for such cases can be represented by the relation;

$$
q^{\prime \prime} \propto h
$$

c. The cooling time for such cases can be represented by the relation;

$$
t \propto\left(\frac{1}{R e}, D^{*}, \frac{1}{S}\right)
$$

\section{ACKNOWLEDGEMENT}

The authors wish to acknowledge The University of Nottingham, Malaysia for the financial support to this research work.

\section{REFERENCES}

[1] A. E. Bergles, Heat transfer enhancement-The encouragement and accommodation of high heat fluxes, J. Heat Transfer(Trans ASME), Vol. 119, pp. 8-19, 1997.

[2] S. Kakaç and A. Pramuanjaroenkij, Review of convective heat transfer enhancement with nanofluids, Int. J. Heat Mass Transfer, Vol. 52(13-14), pp. 3187-3196, 2009.

[3] R. Razgaitis and J. P. Holman, A survey of heat transfer in confined swirl flows, Heat Mass Transfer Processes, Vol. 2, pp. 831-866, 1976.

[4] J. P. Joule, On the Surface-Condensation of Steam, Phil. Trans. R. Soc. Lond., Vol. 151, pp. 133-160, 1861.

[5] S. R. de Farias Neto, P. Legentilhomme and J. Legrand, Finite element simulation of mass transfer in laminar swirling decaying flow induced by means of a tangential inlet in an annulus, Compu. Method Appl. M Eng., Vol. 190(35-36), pp. 4713-4731, 2001.

[6] E. Buckingham, On Physically Similar Systems; Illustrations of the Use of Dimensional Equations, Physical Review, Vol. 4(4), p. 345, 1914.

[7] J. Zhang, H. Lu, L. Zhou and S. Nieh, Simulation of Annular Swirling Turbulent Flows With A New Algebraic Reynolds Stress Model, Numer. Heat Transfer B, Vol. 31(2), pp. 235 - 249, 1997.

[8] M. J. Minkowycz, E. M. Sparrow and J. Y. Murthy, Handnook of Numerical Heat Transfer. Wiley, 2006, New Jersey.

[9] J. Zhang, L. Dong, L. Zhou and S. Nieh, Simulation of Swirling Turbulent Flows and Heat Transfer In An Annular Duct, Numer. Heat Transfer A, Vol. 44(6), pp. 591 - 609, 2003.

\section{NOMENCLATURE}

\section{Latin alphabets}
A
Area, $\mathrm{m}^{2}$
Bs $\quad$ Surface magnetic flux density, $\mathrm{T}$
C Heat capacity, $\mathrm{J} \mathrm{kg}^{-1} \mathrm{~K}^{-1}$
D Diameter, $\mathrm{m}$
D* Ratio of inner diameter to outer diameter
h Heat transfer coefficient, $\mathrm{W} \mathrm{m}^{-2} \mathrm{~K}^{-1}$ 
IIUM Engineering Journal, Vol. 12, No. 6, 2011: Special Issue in Science and Ethics

Kelvin et al.

$\begin{array}{ll}\mathrm{ID} & \text { Inner diameter, mm } \\ \mathrm{k} & \text { Thermal conductivity, } \mathrm{W} \mathrm{m}^{-1} \mathrm{~K}-1 \\ \mathrm{~L} & \text { Length, } \mathrm{mm} \\ \mathrm{m} & \text { Mass, } \mathrm{kg} \\ \mathrm{Nu} & \text { Nusselt number } \\ \mathrm{OD} & \text { Outer diameter, } \mathrm{mm} \\ \mathrm{P} & \text { Pressure, Pa } \\ \mathrm{Pr} & \text { Prantl number } \\ \mathrm{q} & \text { Heat flux, } \mathrm{W} \mathrm{m} \mathrm{m}^{-2} \\ \mathrm{Re} & \text { Reynolds number, } \\ \mathrm{S} & \text { Swirl number } \\ \mathrm{T} & \text { Temperature, } \mathrm{K} \\ \mathrm{t} & \text { Time, } \mathrm{s} \\ \mathrm{U}, \mathrm{V} & \text { Velocity components, } \mathrm{m} \mathrm{s}^{-1}\end{array}$

\section{Greek alphabets}

$\begin{array}{ll}\alpha & \text { Thermal diffusivity of solid, } \mathrm{m}^{2} \mathrm{~s}^{-1} \\ \mu & \text { Dynamic viscosity, Pa s } \\ \rho & \text { Density, } \mathrm{kg} \mathrm{m}^{-3}\end{array}$

\section{Subscript}

$\begin{array}{ll}\text { eff } & \text { Effective } \\ f & \text { Fluid } \\ h & \text { Hydraulic } \\ i & \text { Inner diameter } \\ \text { ini } & \text { Initial } \\ o & \text { Outer diameter } \\ p & \text { Constant pressure } \\ s & \text { Solid }\end{array}$

